\title{
Negative thermal expansion of nanoporous anodic aluminum oxide membranes
}

Cite as: Appl. Phys. Lett. 114, 111901 (2019); https://doi.org/10.1063/1.5074083

Submitted: 21 October 2018 . Accepted: 04 March 2019. Published Online: 18 March 2019

L. Forzani (D) C. A. Ramos (D), E. Vassallo Brigneti (D), A. M. Gennaro (D), and R. R. Koropecki (D)
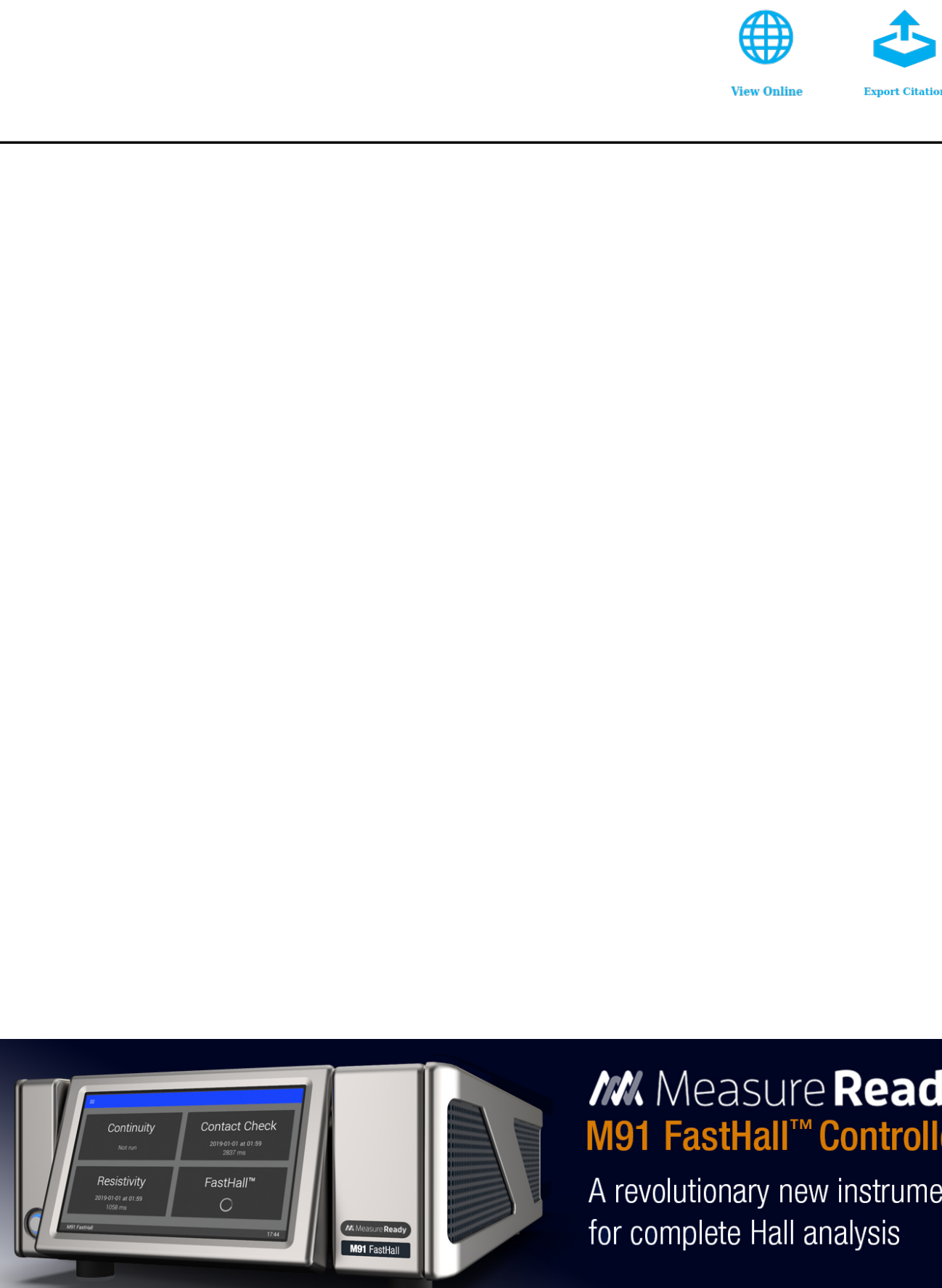

Measure Ready M91 FastHall ${ }^{\text {TM }}$ Controller

A revolutionary new instrument for complete Hall analysis 


\title{
Negative thermal expansion of nanoporous anodic aluminum oxide membranes
}

\author{
Cite as: Appl. Phys. Lett. 114, 111901 (2019); doi: 10.1063/1.5074083 \\ Submitted: 21 October 2018 - Accepted: 4 March 2019 . \\ Published Online: 18 March 2019
}



\section{Forzani, (D) C. A. Ramos, ${ }^{2, a)}$ (D) E. Vassallo Brigneti, ${ }^{3}$ (D) A. M. Gennaro, ${ }^{1,4}$ (D) and R. R. Koropecki i (D)}

\author{
AFFILIATIONS \\ 'Instituto de Física del Litoral, UNL-CONICET. Güemes 3450, 3000 Santa Fe, Argentina \\ ${ }^{2}$ Centro Atómico Bariloche, Comisión Nacional de Energía Atómica, Av. E. Bustillo 9500, R8402AGP, S. C. de Bariloche, \\ Río Negro, Argentina \\ ${ }^{3}$ Departamento de Matemáticas y Física, Instituto Tecnológico y de Estudios Superiores de Occidente, ITESO, \\ Av. Periférico Sur Manuel Gómez Morín 8585, C. P. 45604, Tlaquepaque, Jalisco, Mexico \\ ${ }^{4}$ Departamento de Física, Facultad de Bioquímica y Ciencias Biológicas, UNL. Ciudad Universitaria, 3000 Santa Fe, Argentina \\ ${ }^{a)}$ Author to whom correspondence should be addressed: cramos@cab.cnea.gov.ar
}

\begin{abstract}
We have measured the thermal expansion of Ni nanowires (NWs) electrodeposited into self-organized nanoporous amorphous aluminum oxide (AAO) membranes without an $\mathrm{Al}$ substrate using X-ray diffraction between $110 \mathrm{~K}$ and $350 \mathrm{~K}$. The results indicate an average thermal expansion of the Ni NWs-along the wire axis-of $\bar{\alpha}_{N i N W}=-(1.6 \pm 1.5) \times 10^{-6} \mathrm{~K}^{-1}$. Assuming a bulk-like thermal expansion of the isolated Ni NWs, this result indicates that AAO also has a negative thermal expansion. We estimate the thermal expansion of nanoporous AAO to be $\alpha_{A A O}=-(5 \pm 1) \times 10^{-6} \mathrm{~K}^{-1}$. We show that data obtained previously on the thermal expansion of metallic NWs grown in the nanoporous $\mathrm{AAO}$ may be interpreted as originating from a negative thermal expansion of the matrix.
\end{abstract}

Published under license by AIP Publishing. https://doi.org/10.1063/1.5074083

It is well known that nanostructured materials are characterized by different properties as compared with their bulk counterparts. Characterizing these properties is basic in research and applications. ${ }^{1}$ One of the self-organized nanostructured materials most used is nanoporous anodized aluminum oxide $(\mathrm{AAO}){ }^{2,3}$ To characterize this particular nanomaterial, it is necessary to determine its properties, such as elastic constants, ${ }^{4,5}$ annealing effects, ${ }^{6}$ thermal conductivity, ${ }^{7}$ thermal expansion, ${ }^{8}$ and Poisson ratio ${ }^{9}$ among others. In this work, we focus on an experimental estimate of the thermal expansion of nanoporous AAO using electrodeposited Ni nanowires (NWs) as strain sensors.

Zhang et al. ${ }^{8}$ made a significant effort to measure the thermal expansion of AAO using a modified AFM microscope to determine the thickness change of a $3 \mu \mathrm{m}$ thick membrane of porous AAO grown on a glass substrate. The average $\alpha(T)$, between $300 \mathrm{~K}$ and $400 \mathrm{~K}$, turns out to be about three times larger than the values obtained for bulk alumina. ${ }^{3}$ Also, these values show an increase in $\approx 70 \%$ on going from 300 to $400 \mathrm{~K}$, which may be compared with the bulk alumina change of only $3 \%$ increase in this temperature range. ${ }^{10}$

Other authors have used AAO as templates to electrodeposit nanowires (NWs) of different metals and in-situ XRD to measure the thermal expansion of these embedded NWs. Among these, Xu et al. ${ }^{11}$ measured a near-zero thermal expansion of Ag NWs (considering it from 0 to $650^{\circ} \mathrm{C}$ ). In their analysis, Xu et al. did not take into consideration the AAO matrix in which the Ag NWs were electrodeposited. Instead, they suggest that vacancies in the Ag NWs were responsible for the observed $\alpha \cong 0$ for the NWs, in spite of not reaching the expected bulk value of $\alpha(\mathrm{T})$ of silver even after the samples were annealed up to $800^{\circ} \mathrm{C}$. A similar result was reported when studying $\mathrm{Cu}$ NWs electrodeposited into the AAO templates by Zhou et al. ${ }^{12} \mathrm{Cai}$ et al. $^{13}$ studied Ni NWs by in-situ XRD and EXAFS. By XRD, they measured a thermal expansion coefficient similar to that of bulk $\mathrm{Ni}$, but EXAFS gave a larger value. They explained the results proposing the presence of a $50 \%$ of amorphous Ni in their NW. It should be remarked that in Ref. 13 the Ni NWs were electrodeposited by DC.

The large values for the thermal expansion coefficient of AAO reported by Zhang et al. ${ }^{8}$ are not consistent with the previously reported magnetic anisotropy change observed in Ni NWs grown into the nanopores of AAO, which points out to the fact that AAO has a thermal expansion coefficient smaller than $\mathrm{Ni}$ below room temperature. ${ }^{14-17}$

In this work, we report the thermal expansion coefficient of $\mathrm{Ni}$ NW embedded in the AAO matrix between $110 \mathrm{~K}$ and $350 \mathrm{~K}$. We 
argue that the measured value is mainly determined by the AAO matrix and gives an estimate of the as-prepared nanoporous AAO thermal expansion in this temperature range.

Nanoporous AAO was produced by a two-step anodization process $^{2}$ using $0.3 \mathrm{M}$ oxalic acid at $7^{\circ} \mathrm{C}$ and $40 \mathrm{~V}$. The voltage after the second anodization was decreased exponentially from $40 \mathrm{~V}$ to $8.3 \mathrm{~V}$ in order to decrease the thickness of the insulating barrier layer between the nanoporous $\mathrm{AAO}$ and the $\mathrm{Al}$ substrate to the optimum value of $10 \mathrm{~nm}^{18}$

The Ni filling of the porous structure was made by pulsed electrodeposition using a repeated application of a negative pulse followed by a positive pulse to discharge the capacitor formed by the barrier layer. ${ }^{19}$ The cycle ends with a "dead time" at $0 \mathrm{~V}$ for rearrangement of the electrolyte into the pores. Using SEM, we obtained an estimate of the $\mathrm{Ni}$ NW diameter, $d \cong 40 \mathrm{~nm}$, and an average separation between the wire centers, $D \cong 120 \mathrm{~nm}$. Thus, the area-filling-factor, $f=\frac{\pi}{2 \sqrt{3}}\left(\frac{d}{D}\right)^{2}$, of the $\mathrm{Ni}$ NW with respect to the total area is approximately $10 \%{ }^{20}$

The resulting NW length depends on the electrodeposition conditions and time. In the samples considered here, the Ni NWs are much shorter than the AAO in thickness. Figure 1 shows a lateral and a top view of one of the samples under study.

The aluminum substrate was removed by chemical etching with a $\mathrm{HCl}-\mathrm{CuCl}_{2}$ solution. Thus, the samples under study, typically $\sim 20 \mu \mathrm{m}$ thick, are not subject to the large thermal contraction of the Al substrate.

X-Ray diffraction was performed on a $\theta-2 \theta$ configuration in a PW1710 Philips with a $\mathrm{Cu} \mathrm{K}$. line. A commercial low temperature controller, PAAR TTK-450, was used to fix the temperature, $T$, in the range $110 \mathrm{~K} \leq T \leq 350 \mathrm{~K}$. The samples were set in thermal contact with a $\mathrm{Si}(001)$ wafer by a small amount of vacuum grease covering less than $\approx 5 \%$ of the sample area to decrease possible strain effects on the membrane. After the measurement was performed, the sample was removed unbroken. The Si wafer was used with a double purpose: to eliminate background from the sample holder and to provide with an in-situ thermal expansion reference when the Si (004) reflection condition was met. The sample height was adjusted to be at the required X-ray beam position by properly adjusting a $\mathrm{Cu}$ supplement under the Si wafer. The Si (004) reflection could be eliminated by tilting slightly the sample angular position, which could be adjusted independently of the angular position of the detector $(2 \theta)$. We checked

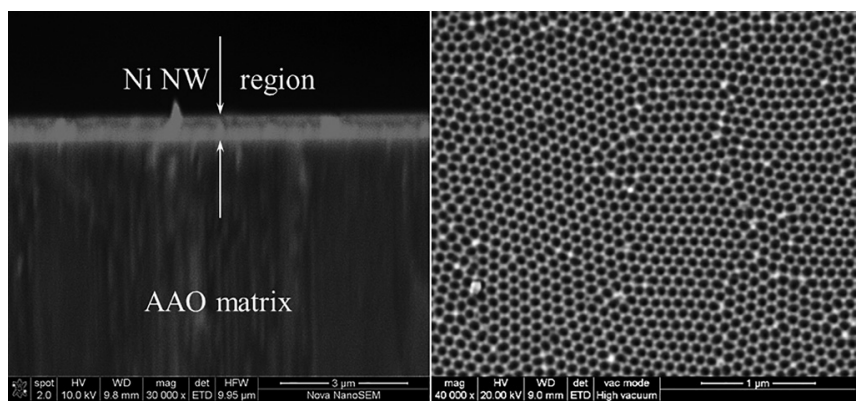

FIG. 1. Left: Lateral view of one of the samples used in this study showing the length of the nanowires. Right: Top view of the same sample. The pores are $\approx 40 \mathrm{~nm}$ in diameter and their center-to-center spacing is $\approx 120 \mathrm{~nm}$, indicating a filling factor, $f \cong 0.1$. that the Ni NW $\theta-2 \theta$ scans were not affected this small tilt $\left(<1^{\circ}\right)$. In Fig. 2, we show a $\theta-2 \theta$ XRD scan from which we deduced that the AAO membrane gives broad peaks associated with its amorphous structure while the Ni NWs show the expected FCC peaks with a lattice parameter $(352.5 \pm 0.4) \mathrm{pm}$, which matches the reported bulk value. Applying Scherrer's formula to the (111) and (220) peak profiles (Fig. 3 and supplementary material), we estimate a crystallite size of 25 and $75 \mathrm{~nm}$, respectively. These magnitudes are similar to what other authors have reported.

In Fig. 3, we present the $\theta-2 \theta$ scans of a sample around the (111) reflection at two different temperatures, $T=125 \mathrm{~K}$ and $T=310 \mathrm{~K}$. The peak line shape was adjusted using non-linear fitting routine with two Lorentzian lineshapes of intensity 2:1 associated with the $\mathrm{CuK}_{\alpha 1}$ and $\mathrm{CuK} \mathrm{K}_{\alpha 2}$ lines plus a linear background. In Fig. 3, we indicate the peak position. The (111) reflection at $T=310 \mathrm{~K}$ is shifted for clarity. The line position was determined within an uncertainty of $0.04^{\circ}$. A similar behavior was obtained from the (220) reflection (supplementary material). The temperature was cycled from room temperature to $T \cong 120 \mathrm{~K}$ and back each time we measured around the $\mathrm{Ni}$ (220), Si (004), and finally Ni (111) peaks. Thus, we claim that the observed behavior is reproducible.

In Fig. 4, we plot the (111) peak positions as a function of temperature. Performing a linear regression of the data between $125 \mathrm{~K}$ and $350 \mathrm{~K}$, we obtain $2 \theta_{111}=(44.405 \pm 0.006)^{\circ}+(1.04 \pm 0.26) \times 10^{-4}(T(K)$ $-300 K)^{\circ} \mathrm{K}^{-1}$. The positive slope in $2 \theta_{111}(d \theta / d T=(0.91 \pm 0.23)$ $\left.\times 10^{-6} \mathrm{rad} / \mathrm{K}\right)$ indicates a small negative thermal expansion coefficient given by

$$
\alpha(T)=-\frac{1}{\tan \theta} \frac{d \theta}{d T},
$$

from where we deduce an average thermal expansion coefficient between $125 \mathrm{~K}$ and $350 \mathrm{~K}$ of $\bar{\alpha}_{N i N W}=-(2.2 \pm 0.6) \times 10^{-6} \mathrm{~K}^{-1}$ for the Ni NW embedded in the AAO matrix.

The Ni (220) Bragg reflection suggests a similar result: $\bar{\alpha}_{N i N W}$ $=-(2 \pm 3) \times 10^{-6} \mathrm{~K}^{-1}$ (supplementary material), albeit with a larger

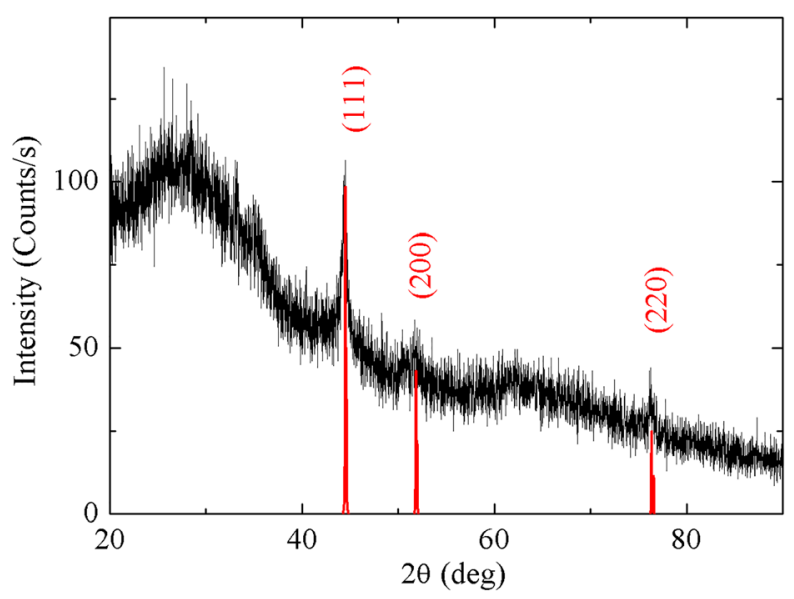

FIG. 2. Room temperature XRD of the Ni nanowires embedded in the porous anodic aluminum oxide template. The Ni peak positions coincide with the expected bulk values. The anodic aluminum oxide matrix shows broad peaks consistent with its amorphous character. 


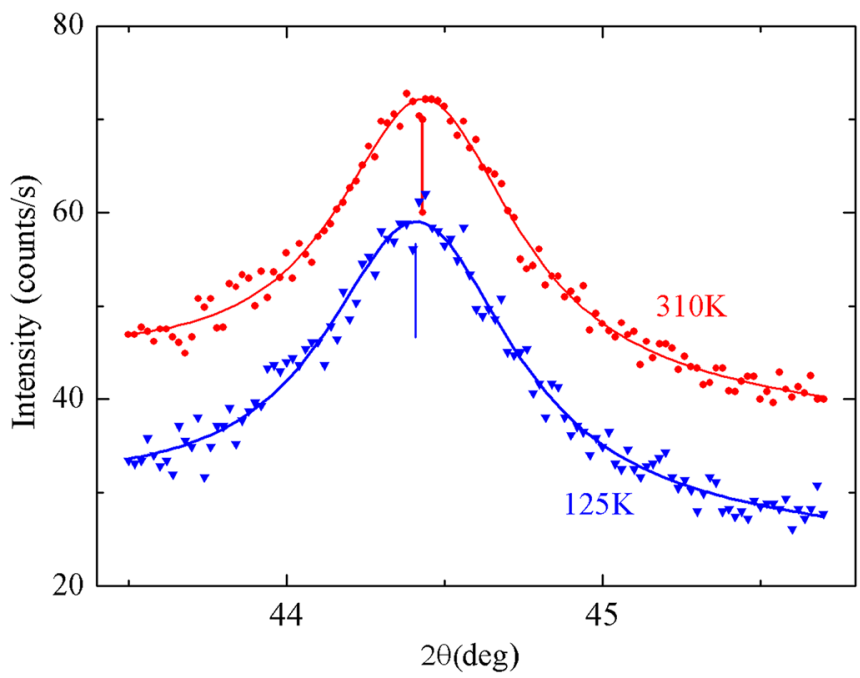

FIG. 3. XRD scan of the (111) Ni NW reflection. The solid line corresponds to the best fit of the data to two Lorentzian lineshapes in a 2:1 intensity ratio as expected from the $\mathrm{K}_{\alpha 1}: \mathrm{K}_{\alpha 2}$ and a linear background. The $310 \mathrm{~K}$ line was shifted upward for clarity.

error. We have considered the effect of systematic errors derived from (a) sample height changes and (b) differences between the sample temperature and the programed temperature (supplementary material). From our experiments, we conclude that $\bar{\alpha}_{N i N W}=-(1.6 \pm 1.5)$ $\times 10^{-6} \mathrm{~K}^{-1}$ in the temperature range of this study.

The average thermal expansion coefficient of bulk Ni between $125 \mathrm{~K}$ and $300 \mathrm{~K}$ is $\bar{\alpha}_{N i}=11.4 \times 10^{-6} \mathrm{~K}^{-1} .{ }^{21}$ For bulk alumina, using the results of Hayashi et al., ${ }^{10} \bar{\alpha}_{\mathrm{Al}_{2} \mathrm{O}_{3}}=4.0 \times 10^{-6} \mathrm{~K}^{-1}$. It is obvious that the Ni NW thermal expansion does not behave either as bulk Ni or as bulk alumina.

There is an additional clue as to what is happening to the Ni NW provided by the magnetic anisotropy associated with the large



FIG. 4. Linear fit of the (111) peak position as a function of $T$. Note that this positive slope indicates a negative thermal expansion of the Ni NW embedded in the AAO matrix. magnetostriction of this material. ${ }^{14,16,22}$ When studying the magnetic behavior of Ni NW embedded in a nanoporous AAO matrix, these authors observed a reduced magnetic anisotropy upon cooling, which is consistent with the anomalous thermal expansion of the Ni NW in the AAO matrix associated with an elongation of the NW upon cooling. However, in these works, the Al substrate was not removed, which has been claimed to be responsible for significant magnetoelastic effects observed. We note that, if the strain parallel and perpendicular to the NW axis were equal, then the magnetostrictive effects on the $\mathrm{Ni}$ NW would compensate. A more detailed study of the magnetostriction effect on the magnetic properties of this system is under way. Magnetoelastic effects are a consequence of the anomalous strains of the Ni NW in the matrix, the latter being caused by the mismatch between the NW and the matrix thermal expansion and elastic properties as we suggest below.

If a perfect bond is assumed between the Ni NW and the AAO matrix, the measured thermal expansion coefficient would correspond to that of the nanocomposite. When considering the mechanical response of aligned fiber composites, Mallick ${ }^{23}$ shows that the composite average thermal expansion can be written as

$$
\bar{\alpha}_{c}=\frac{f E_{N i} \bar{\alpha}_{N i}+(1-f) E_{A A O} \bar{\alpha}_{A A O}}{f E_{N i}+(1-f) E_{A A O}},
$$

where $f$ is the filling factor of the Ni nanowires, and $E_{N i}$ and $\bar{\alpha}_{N i}$ are the Young modulus and average thermal expansion coefficient of $\mathrm{Ni}$ and similarly for the nanoporous AAO. The thermal expansion measured corresponds to the composite under the assumption of elastic deformation and a perfect bond between the Ni nanowires and the AAO matrix $\left(\bar{\alpha}_{c}=\bar{\alpha}_{N i N W}\right)$. A similar result to Eq. (2) was used more recently by Piraux et al. when considering polycarbonate embedded magnetic nanowires and magnetoelastic effects. ${ }^{24}$ Clearly, if $f \rightarrow 0$, then the measured thermal expansion of the composite would correspond to that of the AAO matrix. To account for the Ni modification of the observed thermal expansion of the composite as compared to the AAO thermal expansion, we simply convert Eq. (2) into

$$
\bar{\alpha}_{A A O}=\bar{\alpha}_{N i N W}(1+C)-C \bar{\alpha}_{N i},
$$

where $C=\left[E_{N i} f / E_{A A O}(1-f)\right]$. The Young moduli of Ni and asprepared nanoporous $\mathrm{AAO}$ are $E_{N i}=200 \mathrm{GPa}^{25}$ and $E_{A A O}=147$ $\mathrm{GPa}^{4}$ or $E_{A A O}=114 \mathrm{GPa}^{5}$ from which we obtain an average of $C=(0.17 \pm 0.04)$ where the error considered amounts to a rough $20 \%$ in C. From Eq. (3), we obtain $\bar{\alpha}_{A A O}=-(3.8 \pm 1.9) \times 10^{-6} \mathrm{~K}^{-1}$ for the average thermal expansion of the unperturbed nanoporous AAO matrix along the pore direction. This negative value for the thermal expansion of AAO contrasts with the bulk alumina value which is positive, yet of similar magnitude.

Searching for previously reported thermal expansion of other $\mathrm{NW}$, we found the results of Xu et al. on Ag NWs grown on AAO. ${ }^{11}$ $\mathrm{Xu}$ et al. report an average thermal expansion of the as-prepared $\mathrm{Ag}$ NW of $\bar{\alpha}_{A g N W}=6.35 \times 10^{-9} K^{-1}$ between room temperature and $650^{\circ} \mathrm{C}$. Note that this value is more than three orders of magnitude smaller than bulk Ag: $\bar{\alpha}_{A g}=20.8 \times 10^{-6} K^{-1}$ for the average value between room temperature and $571{ }^{\circ} \mathrm{C}^{26}$ From the reported preparation conditions, which yield a definite pore distance $D$ and the reported pore diameter $d$, we calculate their filling factor $f=0.27 \pm 0.05$ (see supplementary material). Considering a reported Young modulus for 
Ag of $E_{A g}=85 \mathrm{GPa}^{27}$ we derive $C=0.24 \pm 0.06$. From these results, we obtain a corrected thermal expansion for the unperturbed AAO matrix of $\bar{\alpha}_{A A O}=-(5.0 \pm 1.3) \times 10^{-6} K^{-1}$.

When measured by $\mathrm{X}$-ray diffraction above room temperature in $\mathrm{Cu}$ NW electrodeposited into AAO, Zhou et al. ${ }^{12}$ report a nearly zero thermal expansion between room temperature and $300^{\circ} \mathrm{C}$ : for the (220) Bragg diffraction peak using their Fig. 4(c), we obtain $\bar{\alpha}_{C u N W}$ $=(0.0 \pm 0.5) \times 10^{-6} K^{-1}$ for the as-prepared samples. In their text, Zhou et al. show that the $\mathrm{Cu}$ NW diameter is $30 \mathrm{~nm}$. From their AAO preparation conditions, we calculate $f=0.23 \pm 0.05$. Considering $E_{C u}=130 \mathrm{GPa},{ }^{27}$ we obtain $C=0.30 \pm 0.06$. The reported bulk $\mathrm{Cu}$ length change between 577 and $293 \mathrm{~K}$ is $\Delta l / l=0.005$ which would yield an average $\bar{\alpha}_{C u}=17.6 \times 10^{-6} K^{-1}{ }^{26}$ From this, we derive $\bar{\alpha}_{A A O}=-(5.3 \pm 1.1) \times 10^{-6} K^{-1}$.

In the same way, for Fe NWs in the AAO matrix, $\mathrm{Xu}$ et al. ${ }^{28}$ show a slightly negative thermal expansion $\bar{\alpha}_{\mathrm{FeNW}} \approx-0.2$ $\times 10^{-6} \mathrm{~K}^{-1}$ for the data between room temperature and $250^{\circ} \mathrm{C}$. From their work, we estimate $f=0.19 \pm 0.04, C=0.34 \pm 0.07$, which yield $\bar{\alpha}_{A A O}=-(5.1 \pm 1.0) \times 10^{-6} K^{-1}$.

The above arguments assume that NWs behave as bulk material regarding its thermal expansion and elastic properties. When going to sizes comparable to the lattice parameter in one, ${ }^{29}$ two, ${ }^{22}$ or three dimensions, ${ }^{30}$ we may expect these properties to change following finite-size-scaling. ${ }^{31,32}$ We noted that our samples crystallize in the FCC structure with a lattice parameter consistent with bulk values. We also noted that the NW crystallite size is $\sim 100$ lattice parameters. The departure from bulk values for this NW diameter is expected to be very small. Indeed, we were not able to differentiate the lattice parameter from its bulk value.

Recently, Ho et al. ${ }^{33}$ calculated the thermal expansion of isolated ultrathin NW of several FCC metals using molecular dynamics. Although they found negative thermal expansion for some of the metals studied, Ni NWs of diameter $d \cong 5 \mathrm{~nm}$ show a positive thermal expansion coefficient slightly less than the bulk value, and it is expected to approach the bulk value following the finite-size scaling hypothesis.

The magnitude of the finite-size-effect on the magnetic transition temperature of Ni NWs as a function of their diameter was reported to be $\sim 1 \%$ (decrease) for $d=40 \mathrm{~nm}^{22}$ We expect a similar order-ofmagnitude effect on the thermal expansion coefficient of isolated $\mathrm{Ni}$ NW of similar diameter.

Nonetheless, an independent measurement of the thermal expansion of isolated Ni NW and the nanoporous AAO membranes is necessary to corroborate the results deduced in this work using Ni NW as deformation sensors embedded in AAO.

In conclusion, our results on the thermal expansion of the $\mathrm{Ni}$ NW embedded in a nanoporous AAO matrix from $110 \mathrm{~K}$ up to $350 \mathrm{~K}$, as well as previous results on $\mathrm{Ag}-, \mathrm{Cu}-$, and $\mathrm{Fe}-\mathrm{NWs}$ above room temperature, can be interpreted as associated with a negative thermal expansion coefficient of $\mathrm{AAO}$ along the pore direction with an average value of $\bar{\alpha}_{A A O}=-(5 \pm 1) \times 10^{-6} \mathrm{~K}^{-1}$. The origin of the negative thermal expansion pointed by this study is certainly intriguing, and it may be linked to perpendicular motions of atoms or groups of atoms arranged differently from the crystalline state. ${ }^{34}$
See supplementary material for the results of thermal expansion measured on the $\mathrm{Ni}$ (220) Bragg reflection, filling factor determination from anodizing conditions, and systematic error considerations due to the sample height and temperature difference between the programed temperature and sample temperature.

L.F. has a scholarship from CONICET. A.M.G. and R.R.K. are members of the CIC-CONICET. R.R.K. acknowledges support from PICT Grant No. 2014-1683. C.A.R. thanks ITESO invitation as visiting professor (June 2018), PICT 2015\#883 and SePCyT UNCuyo 06/C263.

\section{REFERENCES}

${ }^{1}$ C. Q. Sun, Prog. Mater. Sci. 54, 179 (2009).

${ }^{2} \mathrm{H}$. Masuda and K. Fukuda, Science 268, 1466 (1995).

${ }^{3}$ W. Lee and S. J. Park, Chem. Rev. 114, 7487 (2014).

${ }^{4}$ S. Ko, D. Lee, S. Jee, H. Park, K. Lee, and W. Hwang, Thin Solid Films 515, 1932 (2006).

${ }^{5}$ N. Tsyntsaru, B. Kavas, J. Sort, M. Urgen, and J.-P. Celis, Mater. Chem. Phys. 148, 887 (2014).

${ }^{6}$ M. K. McQuaig, Jr., A. Toro, W. Van Geertruyden, and W. Z. Misiolek, J. Mater. Sci. 46, 243 (2011).

${ }^{7}$ B. Abad, J. Maiz, and M. S. Martin-González, J. Phys. Chem. C 120, 5361 (2016).

${ }^{8}$ X. R. Zhang, T. S. Fisher, A. Raman, and T. D. Sands, Nanoscale Microscale Thermophys. Eng. 13, 243 (2009).

${ }^{9}$ E. VassalloBrigneti, C. A. Ramos, and M. Vázquez (to be published).

${ }^{10}$ H. Hayashi, M. Watanabe, and H. Inaba, Thermochim. Acta 359, 77 (2000).

${ }^{11}$ X. J. Xu, G. T. Fei, W. H. Yu, L. Chen, L. D. Zhang, X. J. Xiao, P. Hao, and B. Y. Wang, Appl. Phys. Lett. 88, 211902 (2006).

${ }^{12}$ W. F. Zhou, G. T. Fei, X. F. Li, S. H. Xu, L. Chen, B. Wu, and L. D. Zhang, J Phys. Chem. C 113, 9568 (2009)

${ }^{13}$ Q. Cai, J. Zhang, X. Chen, Z. Chen, W. Wang, G. Mo, Z. Wu, L. Zhang, and W. Pan, J. Phys.: Condens. Matter 20, 115205 (2008).

${ }^{14}$ S. Kato, H. Kitazawa, and G. Kido, J. Magn. Magn. Mater. 272-276, 1666 (2004).

${ }^{15}$ A. Kumar, S. Fähler, H. Schlörb, K. Leistner, and L. Schultz, Phys Rev. B 73, 064421 (2006).

${ }^{16}$ D. Navas, K. R. Pirota, P. Mendoza Zelis, D. Velázquez, C. A. Ross, and M. Vázquez, J. Appl. Phys. 103, 07D523 (2008).

${ }^{17}$ A. Santos, T. Kumeria, and D. Losic, Trends Anal. Chem. 44, 25 (2013).

${ }^{18}$ K. Nielsch, R. B. Wehrspohn, J. Barthel, J. Kirschner, U. Gösele, S. F. Fischer, and H. Kronmüller, Appl. Phys. Lett. 79, 1360 (2001).

${ }^{19}$ K. Nielsch, A. P. Muller, A.-P. Li, and U. Gösele, Adv. Mater. 12, 582 (2000).

${ }^{20}$ K. Nielsch, J. Choi, K. Schwirn, R. B. Wehrspohn, and U. Gösele, Nano Lett. 2, 677 (2002).

${ }^{21}$ F. C. Nix and D. MacNair, Phys Rev. 60, 597 (1941).

${ }^{22}$ R. López-Ruiz, C. Magén, F. Luis, and J. Bartolomé, J. Appl. Phys. 112, 073906 (2012).

${ }^{23}$ P. K. Mallick, Fiber Reinforced Composites: Materials, Manufacturing and Design (CRC Press, Boca Raton, FL, 2007), Chap. 3.

${ }^{24}$ L. Piraux, G. Hamoir, A. Encinas, J. De La Torre Medina, and F. Abreu Araujo, J. Appl. Phys. 114, 123907 (2013).

${ }^{25}$ H. M. Ledbetter and R. P. Reed, J. Phys. Chem. Ref. Data 2, 531 (1973).

${ }^{26}$ I.-K. Suh, H. Ohta, and Y. Waseda, J. Mat. Sci. 23, 757 (1988).

${ }^{27}$ See www.periodictable.com/Properties/A/YoungModulus.v.html for Cu Young Modulus value.

${ }^{28}$ X. J. Xu, S. F. Yu, S. P. Lau, L. Li, and B. C. Zhao, J. Phys. Chem. C 112, 4168 (2008).

${ }^{29}$ R. Zhang and R. F. Willis, Phys. Rev. Lett. 86, 2665 (2001).

30 J. Wang, W. Wu, F. Zhao, and G.-M. Zhao, Phys. Rev. B 84, 174440 (2011).

${ }^{31}$ M. N. Fisher and M. N. Barber, Phys. Rev. Lett. 28, 1516 (1972).

${ }^{32}$ D. Lederman, C. A. Ramos, V. Jaccarino, and J. L. Cardy, Phys. Rev. B 48, 8365 (1993).

${ }^{33}$ D. T. Ho, S. Y. Kwon, H. S. Park, and S. Y. Kim, Nano Lett. 17, 5113 (2017).

${ }^{34}$ M. T. Dove and H. Fang, Rep. Prog. Phys. 79, 066503 (2016). 